\title{
Smart Fluorescent Probe Strategy for Precision Targeting Hypoxic Tumor
}

Yue Wang and Lingxin Chen*

Cite This: J. Med. Chem. 2021, 64, 2967-2970

Read Online

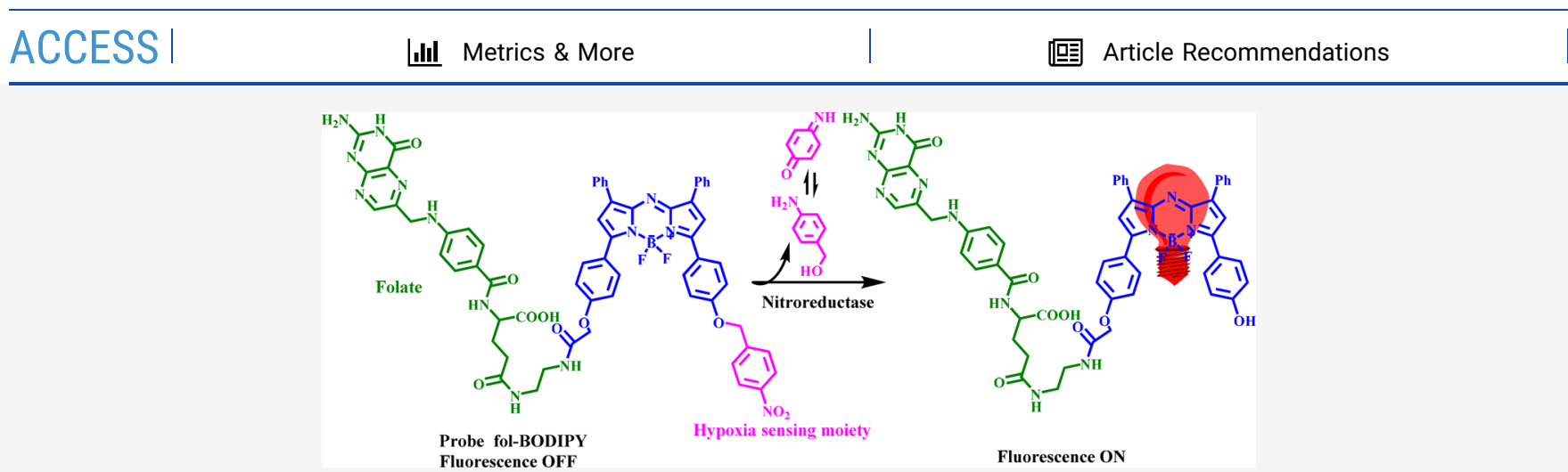

ABSTRACT: Both nitroreductase and the folate receptor are highly expressed in hypoxic tumors. The folate receptor is a potential target for tumors, and nitroreductase can selectively turn on the fluorescence of probes by reducing the nitro group to an amino group. Karan et al. (Karana, S., Cho, M., Lee, H., Lee, H., Park, H., Sundararajan, M., Sessler, J., and Hong, K. Near-infrared fluorescent probe activated by nitroreductase for in vitro and in vivo hypoxic tumor detection. J. Med. Chem. 2021) developed a near-infrared fluorescent probe for hypoxia tumor imaging analysis in vitro and in vivo using folate as the targeting site. This promising strategy may promote advances in precision diagnosis and treatment models of malignant tumors. This Viewpoint looks ahead to new possibilities rendered by their studies.

$\mathrm{M}$ alignant tumors are one of the main hazards to human health. Malignant tumors are extremely aggressive, and the growth, division, and proliferation of tumor cells are incredibly challenging to control, which poses a great challenge to their effective treatment. Surgical resection supplement chemotherapy is the gold standard for tumor treatment. ${ }^{1}$ However, the severe systemic toxicity and side effects of surgical resection and chemotherapy lead to poor prognosis effect. Precision medicine has emerged at a historic moment. Precision medicine has been developed to specifically target and kill tumor cells, which contributes to diagnosis, surgical resection, and prognosis. Therefore, precision medicine has led to advances in treatment models. Early diagnosis and treatment can improve the survival rate of patients by up to $90 \%$. However, the atypical structure and small size of the tumor before carcinogenesis make it difficult to identify tumor lesions. The challenge of precision medicine for tumor treatment is to accurately distinguish the boundary between tumor tissue and healthy tissue.

Accurate targeting of tumors facilitates the development of precision medicine, and it includes passive targeting and active targeting. Passive targeting usually relies on the enhanced permeability and retention effect, and the disadvantage of passive targeting is that it is permeable. Active targeting demands the involvement of targeting units, for example, the small molecule ligands, macromolecular proteins, peptides, and antibodies. Therefore, the key barrier of active targeting is to find the right targeting units. The folate receptor overexpressed on the surface of tumor cells has become a tumor targeting strategy. Folate attaches to the surface of cancer cells and is not cut into the circulation. Folate plays important roles in cell growth, proliferation, and maintenance and has a high affinity for folate receptors on the cell surface $\left(K_{\mathrm{d}}=10^{-10}\right)$, contributing to targeting tumors accurately.

Hypoxia is a typical characteristic of solid tumors mainly due to rapid, exaggerated growth of tumor cells and a disorder of blood vessel tissue resulting in insufficient oxygen supply to the blood. Hypoxia will promote the growth, invasion, and metastasis of malignant tumors that in turn bring about a negative impact on the therapeutic effect of chemotherapy and radiotherapy, leading to poor prognosis and decreased overall survival rate of patients. ${ }^{2}$ Studies have shown that hypoxia

Received: March 10, 2021

Published: March 17, 2021 


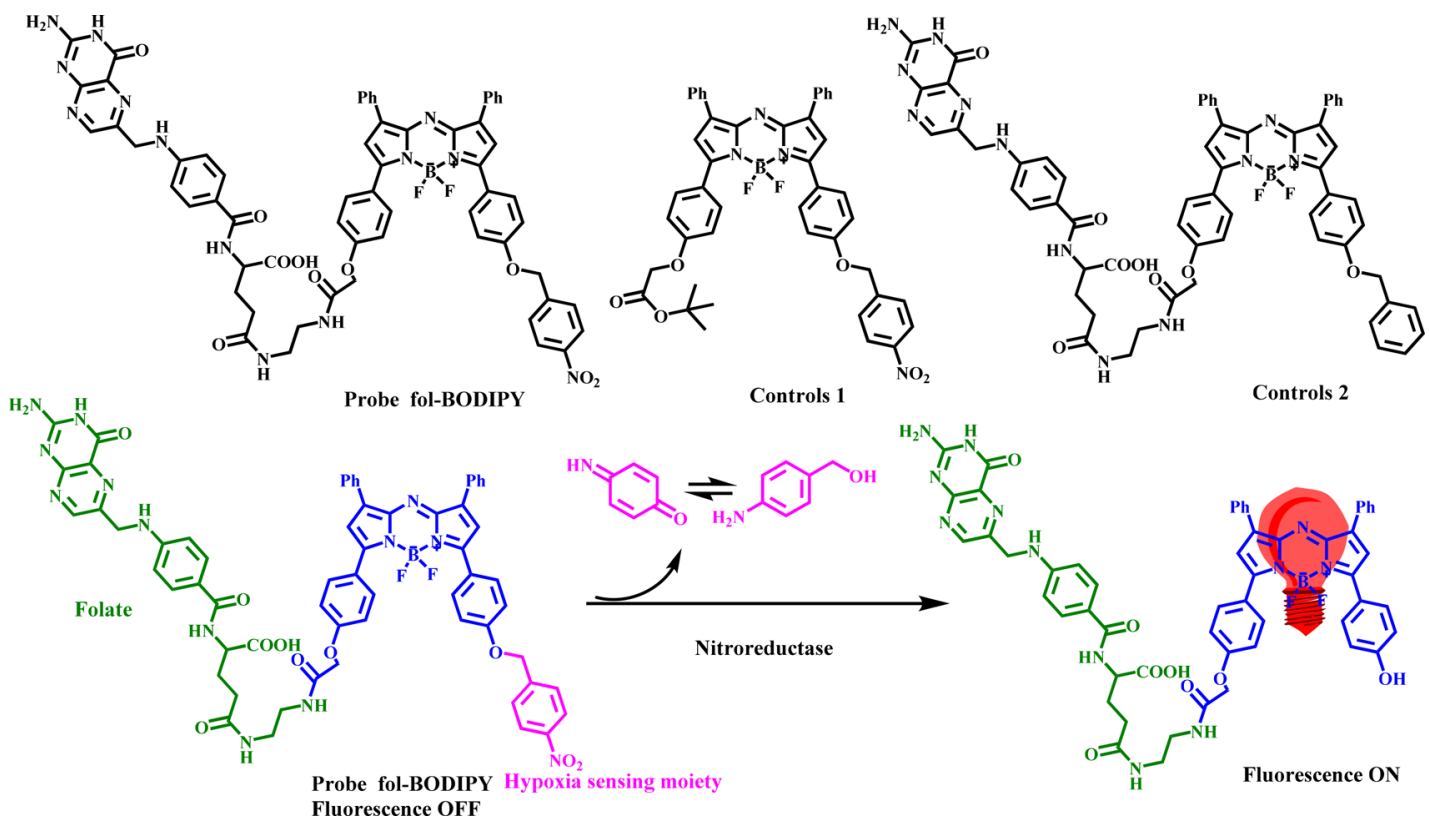

Figure 1. Molecular structure of probe fol-BODIPY, control 1, and control 2 and the proposed mechanism.

leads to overexpression of many tumor-related factors, such as nitroreductase, hypoxia inducible factors $1 \alpha$ (HIF-1 $\alpha$ ), azoreductase, glutathione, and DT-diaphrose. HIF-1 $\alpha$ can cause tumor hypoxic microenvironment deterioration and microvascular abnormality by facilitating metabolic adaptation, angiogenesis, or other pathways. Nitroreductase, azoreductase, and glutathione have been considered as biomarkers for hypoxia detection as well as activators of tumor targeted therapy.

Up to now, the gold standard for clinical detection of tumor hypoxia is to detect the local oxygen partial pressure using an aggressive needle electrode. ${ }^{3}$ Given the invasiveness of the needle electrode, researchers have been working on developing fluorescent probes as powerful tools for hypoxia detection based on the advantage of noninvasiveness, good selectivity, high sensitivity, use in situ, and real-time imaging. Up to now, plenty of nitroreductase-activated fluorescent probes have been widely applied for hypoxia tumor detection. ${ }^{4}$ Nitroreductase is an endogenous flavin-dependent enzyme which is overexpressed in hypoxia tumor cells. With the help of nicotinamide adenine dinucleotide hydrogen $(\mathrm{NADH})$, nitroreductase can transform the nitro group to an amino group, accompanied by the activation of fluorescent signals. ${ }^{5}$ Therefore, developing a nitroreductase-activated fluorescent probe is a fascinating strategy for hypoxia detection.

The work published in this issue of the Journal of Medicinal Chemistry, Karan et al. designed and synthesized a nearinfrared fluorescent probe for hypoxia tumor detection with a folate targeting unit (Figure 1). ${ }^{6}$ They adopted BODIPY as fluorophore due to its advantages in photophysics, such as high fluorescence quantum yield, excellent photostability, and chemical modification. After being connected with the targeting group folate and hypoxia response group $p$-nitrobenzyl ether, the probe fol-BODIPY was obtained. The targeting group folate can enable probe fol-BODIPY aggregate in the tumor, and response group $p$-nitrobenzyl ether can be triggered by nitroreductase, leading to "off-on" fluorescence signal changes under hypoxia. The maximum absorption and emission wavelengths of fol-BODIPY centered at 685 and 730 $\mathrm{nm}$, respectively. The wavelengths located in the near-infrared region (NIR, 650-900 $\mathrm{nm}$ ) can minimize the signal interference caused by tissue absorption and autofluorescence as well as increase the tissue penetration depth. ${ }^{7,8}$ Subsequently, two control probes were synthesized, namely control 1 and control 2 (Figure 1). In contrast to the probe fol-BODIPY, control 1 did not contain targeting group folate, and control 2 did not contain response group $p$-nitrobenzyl ether. Density functional theory studies had shown that probe fol-BODIPY had a lower reaction barrier than control 1 and control 2, indicating that probe fol-BODIPY was more reactive and sensitive toward nitroreductase under hypoxia conditions.

Spectral tests were conducted to investigate the fluorescence response of fol-BODIPY toward nitroreductase under simulative physiological conditions ( $\mathrm{pH}$ 7.4) using $\mathrm{NADH}$ as a cofactor. Probe fol-BODIPY reacted with nitroreductase in the presence of $\mathrm{NADH}$, accompanied by the reduction and departure of the response group $p$-nitrobenzyl ether as well as the activation of fluorescence signals (Figure 1). This response mechanism was confirmed by HR-MS and HPLC chromatogram analysis, and it can react with nitroreductase quickly within $25 \mathrm{~min}$. For nitroreductase detection under simulative physiological conditions, there was a linear relationship between fluorescence intensity and nitroreductase concentration, and the detection limit could reach $1.52 \mathrm{ng} / \mathrm{mL}$. Probe fol-BODIPY exhibited good selectivity toward nitroreductase. Therefore, probe fol-BODIPY was a promising tool for tumor hypoxia detection.

Before cell imaging analysis, cytotoxicity tests of the probe fol-BODIPY and control 1 were conducted. The $\geq 90 \%$ cell viability indicated that both of them had very low toxicity, and then they were applied for hypoxia detection in CT26 and WI38 cells. Probe fol-BODIPY showed bright fluorescence signals under hypoxic ( $1 \%$ oxygen) conditions and weak fluorescence signals under normoxic conditions. However, no fluorescence signal changes were seen from control 1 and control 2 under either anoxic or normoxic conditions. Thus, probe fol-BODIPY can distinguish hypoxia from normoxic effectively. To mimic the hypoxic tumor microenvironments, 


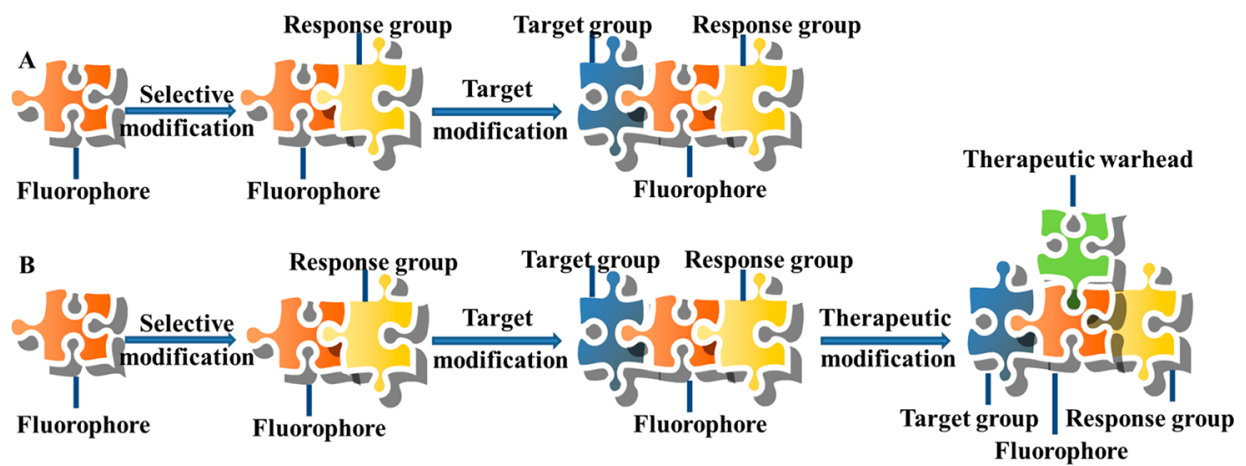

Figure 2. (A) The schematic diagram of probe fol-BODIPY in this article. (B) Optimized schematic diagram for probe design.

3D spheroids were established using folate receptor-negative normal WI38 cells and folate receptor-positive tumor CT26cells. Probe fol-BODIPY exhibited strong fluorescence signals in tumor CT26 cells that overexpressed folate receptor and faint fluorescence signals in normal WI38 cells. However, control 1 and control 2 displayed minimal fluorescence signals. The reasons can be ascribed to the uptake and accumulation of probe fol-BODIPY by tumor cells via folate receptor mediated pathways. Finally, hypoxic imaging was performed in CT26 tumor-bearing nude mice using probe fol-BODIPY and control 1. At $25 \mathrm{~h}$, the signal intensity from probe folBODIPY at the tumor site was 2.4 times stronger than that of control 1; thus, the tumor targeted probe fol-BODIPY containing folate was preferentially ingested and emitted strong fluorescent signals. After sacrifice, tumor tissues and organs (liver, lungs, spleen, and heart) were obtained for imaging analysis, and the fluorescence signal was only observed in the tumor tissue, indicating that the probe fol-BODIPY could effectively target the tumor tissue. Probe fol-BODIPY can promote the precision imaging of tumors; however, the biosafety of probe fol-BODIPY should be evaluated by rigorous medical methods.

To promote oncotherapy and the development of precision medicine, several pieces of advice are given as follows. Most probes like fol-BODIPY contain a fluorophore, target group, and response group, and the schematic diagram of the probe is shown in Figure 2A. In the authors' opinion, probes containing therapeutic warhead may be an intelligent strategy for precision targeted therapy of tumors (Figure $2 \mathrm{~B}$ ), for example, gefitinib is a therapeutic agent for nonsmall cell lung cancer. Furthermore, probes that emit fluorescence in the second nearinfrared window (NIR-II, 900-1300 nm) may be a better choice for imaging analysis given its lower background signal interference. ${ }^{10}$ In addition, chemical stability, biosafety, and pharmacokinetics of fluorescent probes should be carefully examined in the future.

In summary, the study presented by Karan et al. ${ }^{6}$ features not only a fluorescent probe for tumor hypoxia detection but also a strong basis for precision medicine. Tumor targeting units such as the folate receptor, cholecystokin receptor, Glut5 transporter, and carbonic anhydrase IX can accelerate the development of precision medicine in tumor-targeting therapy. New generations of fluorescent probes containing targeting groups and therapeutic agents are important and rapidly developing fields which can accurately achieve drug release. Fluorescence probes for surgical imaging have been developed for use in the clinic, but so far, there have been no clinical trials of small molecule fluorescence therapy. Their research will surely inspire medical scientists and chemists to conceive the next generation of diagnostic and therapeutic agents with better tumor targeting and to promote tumor precision medicine.

\section{AUTHOR INFORMATION}

\section{Corresponding Author}

Lingxin Chen - CAS Key Laboratory of Coastal

Environmental Processes and Ecological Remediation, Yantai Institute of Coastal Zone Research, Chinese Academy of Sciences, Yantai 264003, China; Center for Ocean MegaScience, Chinese Academy of Sciences, Qingdao 266071, China; (1) orcid.org/0000-0002-3764-3515;

Email: lxchen@yic.ac.cn

\section{Author}

Yue Wang - CAS Key Laboratory of Coastal Environmental Processes and Ecological Remediation, Yantai Institute of Coastal Zone Research, Chinese Academy of Sciences, Yantai 264003, China; University of Chinese Academy of Sciences, Beijing 100049, China

Complete contact information is available at: https://pubs.acs.org/10.1021/acs.jmedchem.1c00433

\section{ACKNOWLEDGMENTS}

We thank the National Natural Science Foundation of China (Grant 21976209) and Taishan Scholar Project Special Funding (Grant Ts20190962) for research funding.

\section{REFERENCES}

(1) Gao, M.; Yu, F.; Lv, C.; Choo, J.; Chen, L. Fluorescent chemical probes for accurate tumor diagnosis and targeting therapy. Chem. Soc. Rev. 2017, 46, 2237-2271.

(2) Kwon, D. Y.; Lee, H. E.; Weitzel, D. H.; Park, K.; Lee, S. H.; Lee, C. T.; Stephenson, T.; Park, H.; Fitzgerald, M.; Chi, J.; Mook, R.; Dewhirst, M.; Lee, Y.; Hong, J. Synthesis and biological evaluation of manassantin analogues for hypoxia-inducible factor $1 \alpha$ inhibition. $J$. Med. Chem. 2015, 58, 7659-7671.

(3) Zou, R.; Gong, Q.; Shi, Z.; Zheng, J.; Xing, J.; Liu, C.; Jiang, Z.; Wu, A. A ZIF-90 nanoplatform loaded with an enzyme-responsive organic small-molecule probe for imaging the hypoxia status of tumor cells. Nanoscale 2020, 12, 14870-14881.

(4) Wu, X.; Shi, W.; Li, X.; Ma, H. Recognition moieties of small molecular fluorescent probes for bioimaging of enzymes. Acc. Chem. Res. 2019, 52, 1892-1904.

(5) Li, Y.; Sun, Y.; Li, J.; Su, Q.; Yuan, W.; Dai, Y.; Han, C.; Wang, Q.; Feng, W.; Li, F. Ultrasensitive near-infrared fluorescenceenhanced probe for in vivo nitroreductase imaging. J. Am. Chem. Soc. 2015, 137, 6407-6416. 
(6) Karan, S.; Cho, M.; Lee, H.; Lee, H.; Park, H.; Sundararajan, M.; Sessler, J.; Hong, K. Near-infrared fluorescent probe activated by nitroreductase for in vitro and in vivo hypoxic tumor detection. J. Med. Chem. 2021, DOI: 10.1021/acs.jmedchem.0c02162.

(7) Wang, Y.; Zhang, L.; Chen, L. Glutathione peroxidase-activatable two-photon ratiometric fluorescent probe for redox mechanism research in aging and mercury exposure mice models. Anal. Chem. 2020, 92, 1997-2004.

(8) Fan, L.; Pan, Y.; Li, W.; Xu, Y.; Duan, Y.; Li, R.; Lv, Y.; Chen, H.; Yuan, Z. A near-infrared fluorescent probe with large Stokes shift for visualizing and monitoring mitochondrial viscosity in live cells and inflammatory tissues. Anal. Chim. Acta 2021, 1149, 338203.

(9) Song, X.; Han, X.; Yu, F.; Zhang, X.; Chen, L.; Lv, C. Polyaminetargeting gefitinib prodrug and its near-infrared fluorescent theranostic derivative for monitoring drug delivery and lung cancer therapy. Theranostics 2018, 8, 2217-2228.

(10) Dou, K.; Feng, W.; Fan, C.; Cao, Y.; Xiang, Y.; Liu, Z. Flexible designing strategy to construct activatable NIR-II fluorescent probes with emission maxima beyond $1200 \mathrm{~nm}$. Anal. Chem. 2021, 93, 40064014. 